\title{
Design of Crime Spatio-temporal Analysis System Based on the Police Workflow
}

\author{
Dong Cai ${ }^{1,2,}$, Man $\operatorname{Lin}^{1, b}$ \\ ${ }^{1}$ Public Security Management Department, Jiangsu Police Institute, Nanjing, China \\ ${ }^{2}$ Key Laboratory of Geographic Information Technology of Public Security Ministry, Changzhou \\ City Public Security Bureau, Changzhou, China \\ atsaidong@126.com, blin-man@163.com
}

\begin{abstract}
According to crime spatio-temporal analysis application requirements, this paper researches on the key technology of crime spatio-temporal analysis. With the key technology, crime spatio-temporal analysis system based on the police workflow is designed to provide various analysis functions, such as crime hotspot analysis, a series of crime cases temporal regional impact, criminal residence prediction, and relevance control analysis. The application of crime spatiotemporal analysis system is verified by the typical city practice.
\end{abstract}

Keywords: Crime Spatio-temporal Analysis, Police Workflow, System Design.

\section{Introduction}

Although the information construction in our country has obtained great development, but compared to the police work requirements now, police service informatization construction still appears the relative lag [1-2]. Because of complexity and the lack of connection between each function module in the past the police management system, the police face many problems in the process of system operation [3]. How to realize the connection between systems has become the key to solve the problem of police management. Under this background, this research designs a crime spatio-temporal analysis system based on the police workflow which meets policing management demands. The system design is aim to combine each module of crime spatio-temporal analysis together, so that the routine work of police can be carried out by the workflow.

\section{Workflow Analysis of Crime Spatio-temporal Analysis System}

\subsection{Application Requirement Analysis}

First is the need of work of crime analysis. Crime analysis researchers have developed specialized subject of criminal geography, such as environmental determinism, criminal offence, environmentalism, crime location theory and etc. are proposed recently [4]. All these theories have been a number of practical applications in foreign countries [5]. And also, there are special crime analysts use crime space analysis tool to analyze all kinds of police data for the police department, which can provide the decision information. But in the domestic public security department, because of the lack of professional crime analysis tool, all these theories above have stayed at the theoretical level and macro guidance. At present, in the case of processing department, the policemen can't draw a scientific conclusion through calculation and quantitative analysis. Instead they still use the method of artificial experience and qualitative analysis, although they already know the importance of geographical factors. Because they unable to make effective use of the geographical factors, which can lead to the larger defects in crime analysis.

Second is the need of the further development of police geographical information technology. Usually geographic information technology is used to integrated management and data visualization, and spatial analysis ability is also one of their most important advantages [6]. Through analysis methods such as spatial statistics, spatial clustering, spatial correlation, spatial overlay and etc. the spatial regularity of the data can be effectively analyzed, thus inside information in the data can be obtained, which will give full play to the potential value of the data. Recently further development of 
PGIS platform needs a larger breakthrough in spatial analysis. Otherwise it will restrict the application effect of PGIS and actual combat ability.

Third is the need of study and judge in the public security work. At present there are a large number of spatio-temporal crime data in existing system of the public security organs, in which there are a large number of location information about cases, personnel, goods. From the existing function in recent system, most of dealing with this kind position information are still focus on a simple query positioning function [7-9]. For example, many kinds of management object trajectory information, such as spatial correlation information, regional hot spots and so on, have failed to effectively carry out the analysis and data mining, which restricts the application effect of crime analysis. So, researches about temporal and spatial analysis technology should be developed based on the existing workflow information, so that the analysis capability of public security information system will be improved.

\subsection{Workflow Analysis}

In the crime spatio-temporal analysis system, the city can be divided into different levels of the spatial grid Council, which is based on bureau, police station, police area etc. [10]. On this basis according to the spatial information of all kinds of typical cases, such as Robbery, theft, can be placed in the related spatial grid Council. Then different temporal and spatial scales characters can be studied by this way. And also weighted statistics can be done according to the precise location of the cases occurred in recent years so that the dynamic statistical model about different types cases will be obtained. These models can reflect the evolution regularity of crime in the different regions and different period of time, which can establish the overall workflow process characterization in small and medium-sized city.

In the workflow analysis, a dynamic evaluation index system of city crime space has to be established. Then quantify the city police cases according to the dynamic evaluation index system, so the general illegal indicator of city bureau can be decomposed into precinct, police station and police responsibility area on the basis of crime space model. The evaluation index in different levels of the spatial grid Council can be constructed. According to the long-term, medium-term and short-term illegal control objectives can help determine the current illegal status, and find hot case and case development trend, which will guide the daily work of policemen.

\section{Analysis System Design of Crime Spatio-temporal Analysis System}

\subsection{The System Architecture Design}

The system uses the $\mathrm{B} / \mathrm{S}$ structure pattern, which is divided into three layers of structure. The three layers includes data layer, service layer and application layer, which will provide safe and reliable security system. The data layer is divided into two levels. The first is service data, which includes household registration, public order, and information communication etc. Through data extraction, cleansing, transformation, and periodically service data collection from database, the system can analyze regularly to form personnel relation database, vehicle trajectory database etc. All the data above will summary to the crime analysis database, and regularly update the crime analysis formation into crime analysis data warehouse.

The service layer provides the macroscopic analysis services and empirical study services. The macroscopic analysis services focus on the macro data analysis, which including the data source management, model establishment and calculation results creation, rendering scheme management etc. Empirical study services mainly analysis the case or a series of cases, and cartography or aided crack criminal case, and then show related information for crime spatio-temporal analysis.

The application layer provides analysis services based on the service layer, which aims to realize the actual combat application functions. The functions include regional impact, relationship analysis, relevance control, trend prediction, control of crime hot spot analysis and etc. 


\subsection{Function Design}

The crime spatio-temporal analysis system targeted designs hot spot analysis, trend analysis, informatic Tupu analysis, intelligence analysis, relationship analysis, regional impact analysis, relevance control analysis and so on.

(1) Hot spot analysis. Through a number of cases on particular category and in the selected time period, the spatial location information of these selected cases will be deeply studied, in order to determine the concentration regional of crimes (i.e. crime hotspots). According to the spatial distribution of the illegal data, a high incidence area of illegal cases will be formed. Hot spot analysis is essential for the macroscopic analysis method of crime spatio-temporal analysis, which has the practical significance.

(2) Crime trend analysis. Trend analysis mainly researches on the police intelligence changes during a specified period of time. According to the illegal situation during a certain period of time, trend charts are made to show the illegal trends directly within the scoping or the police unit, which will provide support for macro decision of leadership.

(3)Informatic Tupu analysis. The analysis appears crime changes between different time and area according to various types of crime ratio, time distribution of crime. According to the illegal conditions in a certain region for a period of time, the change characters of illegal cases between different period (month, quarter, or custom time) can be presented visualization.

(4)Intelligence analysis. Intelligence analysis is aim to give an illegal emotion study report. The illegal emotion study report gives a macroscopic show of illegal distribution on the basis of spatial standard deviation and hot spot map, and also realizes the illegal statistical analysis according to the spatial grid Council, completes illegal emotion study report daily and weekly. The report will pay close attention to the different illegal cases comparison and the illegal prediction.

(5) Regional impact analysis. The regional impact analysis mainly focuses on temporal impact analysis, which is based on the implicit personnel social relations between different crime cases. When a series of crime cases happen, the policemen can get the case clues from the impact analysis between a plurality of personnel information in the crime surrounding area, which can help policemen obtain macroscopic crime trends and crime clues of specific case.

\subsection{Database Design}

The data type of crime spatio-temporal database include basic geographic data, vehicle management data, 110 alarm data, plan inference data, population data, address data, belongings data, illegal cases data, and other related data etc. The database model uses the relational model, for there are numbers of data types in the crime spatio-temporal analysis system, and the relationships between these data types are more complicated. Taking population data as the core, illegal cases and dynamic public security workflow data as analysis sources, GIS data and illegal statistics data as the secondary data, the crime spatio-temporal analysis system can analysis the relationship in two dimensions of time and space between these data above. The analysis result will be used directly in the subsequent analysis[11].

The crime spatio-temporal database includes the relation table, spatial table and mass data. Traditional data tables store relational model in the database. But the number of data in spatiotemporal analysis system reaches hundreds of millions, which can reach the number of $1 \mathrm{~TB}$. Because of this, the traditional relational database is very difficult to satisfy the high performance of query and analysis requirements. So the spatio-temporal analysis system in this paper uses mixed intermediate database storage policy which is suitable for the application above. The mixed intermediate database storage policy is based on massive distributed storage framework, which realizes the storage and management of large data. And the mixed intermediate database storage policy based on KV data uses special high-performance data index technology, which can support billion level amount of data access and parallel implementation of data capacity expansion. 


\section{Conclusion and Prospect}

\subsection{Conclusion}

This paper establishes an actual combat spatio-temporal analysis system taking Changzhou City of Jiangsu Province as a typical region, which realizes the processed management of spatio-temporal analysis. The system realizes the intelligent comparison analysis and relationship analysis between amount of illegal cases, which riches judge analysis method, and promotes PGIS platform from visualization to study and judge analysis.

\subsection{Prospect}

At present, the domestic crime analysis mainly pauses in the theory and the macro guidance level, which is lack of special spatio-temporal analysis system to help criminal investigation in public security departments. Although this paper has made some exploration on crime spatio-temporal analysis field, but there are still some problems in the research. For example, how to combine the spatio-temporal analysis with specific public security workflow, and make the research go further and deeper, is the urgent problem to solve. Then designing a nationwide general special spatiotemporal analysis system with high standard and practical ability is the research focus in the future. All the future researches will improve the detection efficiency of public security departments, which is the urgent need of public security practice.

\section{Acknowledgements}

This work was financially supported by a Project Funded by the Priority Academic Program Development of Jiangsu Higher Education Institutions, the Theory and the Soft Science Project of Ministry of Public Security No. 2018LLYJJSST053, the Natural Science Foundation of Jiangsu Province No. BK20141033, the University Science Research Surface Project of Jiangsu Province No. 17KJB170005, Green seedling project of JSPI No. JSPI2018QM, Research and innovation team of JSPI No. 2018SJYTD12 and The Natural Science Project of JSPI No. 2017 SJYZZ01.

\section{References}

[1]. Wang Chao, Zhao Wenji, Zhou Daliang. Research and Design of Crime Analysis System Based on GIS[J]. Journal of Capital Normal University (Natural Science Edition). 2010, 31(3): 47-52.

[2]. Xie Xiaozhuan. The Principle, Mode and Application of Comparative Analysis of Crime Temporal and Spatial Intelligence[J]. Journal of Chinese People's Public Security University (Social Sciences Edition). 2012, (2): 86-91.

[3]. Cai Dong, Gao Chao. Design of Urban Emergency System Based on 3S Spatial Information Technology[J]. Applied Mechanics and Materials. 2014, 6707-6710.

[4]. Sun Fenghua, Li Shitai and Huang Liping. AUTHENTIC PROOF STUDY ON CRIMINAL GEOGRAPHICAL LAWS IN CHINA AND FOREIGN COUNTRIES[J]. Human Geography. 2006, (5): 14-18.

[5]. Harries.K D. The Geography of Crime and Justic[M]. New York: McGraw Hill.1974: 38-49.

[6]. Fahui Wang, Geographic Information Systems and Crime Analysis[M]. Idea Group Publishing. 2004:197-209.

[7]. Wenxue Cai, Guangwen Chen, Qilin Wang, et al. Research on Emergency command system architecture. China Science and Technology Information[J]. 2013, (8): 174-175.

[8]. Jiaowan Zhang. Design and implementation of city emergency response system based on spatial information service[D]. University of Electronic Science and technology, 2013. 
[9]. Ling Zhang. Study on the construction of emergency management system of Huizhou City[J]. Technology Innovation and Application, 2014, (3): 268.

[10]. Smith. T. S. Inverse Distance Variations for the Flow of Crime in Urban Areas[J]. Social Forces. 1976, 25(4): 16-23.

[11]. Francis FAJEMIROKUN, O. ADEWALE, Timothy IDOWU, Abimbola OYEWUSI, Babajide MAIYEGUN. A GIS Approach to Crime Mapping and Management in Nigeria: A Case Study of Victoria Island Lagos[R]. Nigeria: XXIII FIG Congressm, 2006. 